\title{
El turismo CAVE como herramienta para la divulgación del patrimonio y el desarrollo sustentable en el Geoparque aspirante Huasteca Potosina
}

\section{CAVE tourism as a tool for the dissemination of heritage and sustainable development in the Huasteca Potosina Aspirational Geopark}

\author{
DOI: $10.46932 / \mathrm{sfjdv2n5-075}$
}

Received in: Oct 1st, 2021

Accepted in: Dec 30th, 2021

\section{Irma Brígida Suárez Rodríguez}

Maestría en Mercadotecnia de Negocios Turísticos

Universidad Autónoma de San Luis Potosí, Facultad de Estudios Profesionales Zona Huasteca

Romualdo del Campo No. 501 Fracc. Rafael Curiel Cp. 79060 Ciudad Valles, SLP. México

E-mail: irma.suarez@uaslp.mx

\section{Griselda Meraz Acevedo}

Doctoranda en el Programa de Gestión Estratégica de Negocios (UAT-PNPC) Universidad Autónoma de San Luis Potosí, Facultad de Estudios Profesionales Zona Huasteca Romualdo del Campo No. 501 Fracc. Rafael Curiel Cp. 79060 Ciudad Valles, SLP. México

E-mail: griselda.meraz@uaslp.mx

\author{
Alejandro Gutiérrez Hernández \\ Doctorante en Filosofía \\ Universidad Autónoma de San Luis Potosí, Facultad de Estudios Profesionales Zona Huasteca \\ Romualdo del Campo No. 501 Fracc. Rafael Curiel Cp. 79060 Ciudad Valles, SLP. México \\ E-mail: alejandrogh@uaslp.mx
}

\section{Juan Luna-Kelser}

Master of Tourism Administration

The George Washington University, International Institute of Tourism Studies GW School of Business, Duquès Hall, 2201 G Street, Washington, D.C. 20052

\section{RESUMEN}

Con el continuo desarrollo de la industria del turismo en el mundo, los destinos están tratando de diferenciarse para aumentar su competitividad global. Un creciente nicho de mercado que puede aportar grandes beneficios a los territorios en este sentido se identifica como el Turismo CAVE: Científico, Académico, Voluntariado y Educación (SAVE Tourism).1 Las actividades basadas en este segmento aportan beneficios a la comunidad local a través del aumento de los ingresos como consecuencia del incremento de estadía de turistas y mayor gasto, generando nuevas oportunidades de desarrollo socioeconómico o sea, mayor empleo. Además, este tipo de turismo contribuye a generar fondos para la conservación del patrimonio ambiental y un mayor compromiso con la preservación de la cultura local, lo cual eleva y refuerza la conciencia social sobre cuestiones ambientales, culturales y mejores prácticas. En el caso del territorio que conforma el Geoparque aspirante Huasteca Potosina, a través de un estudio realizado por la Universidad Autónoma de San Luis Potosí en los municipios de Aquismón, Ciudad Valles y Xilitla, en colaboración con la George Washington University han surgido recomendaciones para este

\footnotetext{
${ }^{1}$ SAVE por sus siglas en inglés (Scientific, Academic, Volunteer and Educational)
} 
segmento del mercado turístico, a fin de ayudar a mejorar la experiencia del turismo local y crear oportunidades de desarrollo para el sector turístico y las comunidades locales.

Palabras clave: Turismo CAVE, Geoparque, Patrimonio Cultural y Natural, Desarrollo Sustentable.

\begin{abstract}
With the continuous development of the tourism industry in the world, destinations are trying to differentiate themselves in order to increase their global competitiveness. A growing niche market that can bring great benefits to territories in this regard is identified as CAVE Tourism: Scientific, Academic, Volunteer and Education (SAVE Tourism). Activities based on this segment bring benefits to the local community through increased income as a result of increased tourist stays and higher spending, generating new opportunities for socio-economic development, i.e., increased employment. In addition, this type of tourism contributes to generating funds for the conservation of environmental heritage and a greater commitment to the preservation of local culture, which raises and reinforces social awareness of environmental and cultural issues and best practices.

In the case of the territory that makes up the Huasteca Potosina Aspiring Geopark, through a study conducted by the Universidad Autónoma de San Luis Potosí in the municipalities of Aquismón, Ciudad Valles and Xilitla, in collaboration with the George Washington University, recommendations have emerged for this segment of the tourism market to help improve the local tourism experience and create development opportunities for the tourism sector and local communities.
\end{abstract}

Keyword: CAVE Tourism, Geopark, Cultural and Natural Heritage, Sustainable Development.

\title{
1 INTRODUCCIÓN
}

La Huasteca Potosina, una de las cuatro regiones en las cuales se divide el estado de San Luis Potosí, México, posee un patrimonio geológico excepcional, una rica biodiversidad y un importante legado cultural. Esta región cuenta con una clara vocación para aprovechar el turismo como una estrategia para el desarrollo sustentable.

La Universidad Autónoma de San Luis Potosí (UASLP), a través de la actual Facultad de Estudios Profesionales Zona Huasteca (FEPZH), y de su Cuerpo Académico "Estudios del Desarrollo Humano y la Sustentabilidad" ha desarrollado estudios encaminados a presentar una solicitud para lograr la designación de Geoparque Mundial de la UNESCO a seis municipios de la Huasteca Potosina. Los líderes locales del gobierno, el sector privado y los círculos académicos prevén que la designación ayudará a esta región a diferenciarse en el mercado turístico tanto a nivel nacional como internacional, lo cual, en última instancia, se traduce en generar más beneficios para las economías locales que pueden ayudar a crear nuevos puestos de trabajo, reducir la pobreza y conservar y preservar el patrimonio geológico único de la región, así como su rica cultura.

De acuerdo con la Organización de las Naciones Unidas para la Educación, la Ciencia y la Cultura (UNESCO 2020): 
Los Geoparques Mundiales de la UNESCO son espacios geográficos unificados, donde los sitios y paisajes de alcance geológico internacional se gestionan de acuerdo con un concepto global de protección, educación y desarrollo sostenible. Favorecen la toma de conciencia de la geodiversidad y promueven mejores prácticas de protección, educación y turismo. Junto con los sitios del Patrimonio Mundial y Reservas de la Biosfera, los Geoparques forman una gama completa de herramientas para el desarrollo sostenible y contribuyen a la consecución de la agenda 2030 (ODS) a través de la combinación de perspectivas globales y locales.

El proyecto Geoparque Huasteca Potosina se considera como una propuesta innovadora de gestión para este territorio huasteco, que promueve una nueva visión del entorno, a través de la valoración, preservación, conservación y divulgación del patrimonio geológico, natural y cultural, así como la puesta en valor para promover un desarrollo sostenible de las comunidades locales.

Reconocer el potencial del patrimonio abre oportunidades para impulsar el desarrollo socioeconómico de los habitantes. Por ende, el Turismo CAVE se considera como un componente integral del proceso de solicitud de Geoparque Global, que apunta a mejorar la planeación del turismo, para que dicho sector se desarrolle de una manera sustenable que beneficie a la comunidad, la cultura y el medio ambiente local. Como parte de su iniciativa de territorio aspirante a Geoparque (Aspiring Geopark), la UASLP se alió con la George Washington Univeristy (GWU) para llevar a cabo una evaluación del desarrollo turístico sustentable en tres municipios de la región: Aquismón, Ciudad Valles y Xilitla.

\section{MARCO CONCEPTUAL}

Al turismo se le considera un sector relativamente "joven" que ha tenido un desarrollo vertiginoso a partir de 1950, para ese año 25 millones de personas viajaron alrededor del mundo, en 2019 se registraron 1,500 millones de viajeros internacionales (OMT 2020).

Países con economías desarrolladas compiten por el turismo con países de economías emergentes y viajar se convierte casi en una necesidad básica. Como expresa García (2007) "la humanidad globalmente considerada ha optado por ser nómada, eliminando el sentido de territorialidad".

Los gobiernos de diversos países han promovido al turismo como un motor para el desarrollo, pues este genera una importante derrama económica. El turismo para México es una de las bases económicas; de acuerdo con cifras de la Secretaría de Turismo, el sector aportó en 2019 el 8.9 por ciento del Producto Interno Bruto Nacional y contribuyó con más del 9 por ciento de los empleos directos e indirectos (SECTUR 2020).

A pesar de los resultados habitualmente positivos del turismo, no debemos olvidar los aspectos negativos que la experiencia de los sitios turísticos alrededor del mundo ha comprobado que trae consigo, como menciona Jafari (2005) “costos económicos, ambientales y socioculturales”. 
Por lo tanto, es necesario diseñar estrategias que permitan el desarrollo del sector bajo un marco de sustentabilidad, concepto que se ha convertido en uno de los requisitos para atraer a turistas responsables. De acuerdo a Márquez (2004) es una nueva forma de planificar, ofertar y comercializar el producto turístico, fundamentado en principios colectivos éticos para el manejo racional y responsable de los recursos naturales y culturales, cuyos beneficios económicos mejoren la calidad de vida de todos los sectores involucrados. Un turismo basado en el respeto al ambiente, en la calidad más que en la cantidad, en la sostenibilidad a mediano y largo plazo más que en la explotación de recursos a corto plazo (Vidal y Márquez, 2007; Chávez, Andrade y Espinoza, 2013; Pastor, 2014).

En este sentido, el Programa de Geoparques Globales de la UNESCO ofrece a los territorios un marco de gestión para aumentar la conciencia de la diversidad geológica y promover las mejores prácticas de protección, educación y turismo. "Los Geoparques promueven el desarrollo económico local sostenible, principalmente a través del geoturismo" (UNESCO 2020).

Para hablar de Turismo CAVE (Científico, Académico, Voluntariado, Educación) resulta entonces necesario definir el concepto que fue desarrollado en el año de 2003 por el equipo de estudiantes de turismo de postgrado de la Universidad de George Washington (Master's Program in Tourism Administration - Consulting Practicum) como una estrategia para aumentar las visitas a la Costa Norte de Honduras. $^{2}$ Las actividades basadas en este segmento aportan beneficios a la comunidad local a través del aumento de los ingresos como consecuencia del incremento de turistas y nuevas oportunidades de empleo. Además, refuerza la conciencia social sobre cuestiones ambientales y las mejores prácticas, lo que puede dar lugar a un impacto positivo y duradero en el medio ambiente y la cultura.

El mercado CAVE representa un segmento creciente de la industria mundial del turismo que difiere en varios aspectos clave de las formas más convencionales de turismo. Representa una amplia gama de turistas que viajan a un destino para dedicarse a la investigación, el aprendizaje o el turismo de voluntariado.

Por definición, aquellos atraídos al turismo CAVE suelen estar grupo de turistas interesados en actividades que pueden aprovecharse fácilmente para impulsar la preservación del patrimonio natural y cultural, así como una mejoría en las condiciones de vida de los lugareños (SAVE Travel Alliance, s.f.). Las actividades de CAVE afectan no solo a las economías locales, sino que también conllevan beneficios económicos a largo plazo a nivel nacional y local mediante el fomento de las capacidades de las instituciones educativas, científicas y culturales.

Los viajes científicos son aquellos que incluyen experiencias que ofrecen a los visitantes la oportunidad de participar en proyectos de investigación. Quienes típicamente participan en este tipo de

\footnotetext{
${ }^{2}$ SAVE in Campeche - SAVE Travel Alliance. (n.d.). Fuente: http://www.save-travel.org/destinations/campeche-mexico/about/
} 
actividades invierten su tiempo libre acudiendo a sitios remotos del mundo para realizar acciones de investigación, el seguimiento de la vida silvestre y la fauna o aumentando su conocimiento en prácticas de conservación, por mencionar algunas. El propósito de sus viajes es contribuir al desarrollo científico a nivel mundial y para adquirir el conocimiento local. Apoyan a destinos con necesidades de asistencia adicional fuera de sus comunidades, generando beneficios económicos y contribuyendo a la investigación, la protección y conservación del patrimonio de las regiones. ${ }^{3}$

Los viajes académicos incluyen a estudiantes y profesores de la enseñanza superior y postgrado que son impulsados por el deseo de participar en experiencias de viaje que incluyen una interacción directa con la naturaleza, la cultura y la población local, de manera que promuevan el conocimiento y la comprensión de los destinos. A través de estos viajes se pretende impulsar la economía del destino. Estos viajeros pasan más tiempo (una media de 53 días) y gastan más dinero que el turista medio en negocios locales (en promedio USD\$1,000-USD\$6,000). Según el Instituto de Estadística de la UNESCO, el número de estudiantes que cursan estudios en el extranjero sigue creciendo: en 2013, más de 4,1 millones de estudiantes fueron a estudiar al extranjero, frente a los 2 millones en 2000, participando adicionalmente en la promoción de la cultura y el desarrollo sostenible. ${ }^{4}$

El turismo voluntario o voluntariado es aquel en el que los viajeros acuden de voluntarios en las comunidades locales, con la oportunidad de sumergirse en una comunidad mientras retribuye a la misma proporcionando una variedad de servicios tales como la enseñanza de otros idiomas en los centros de aprendizaje o como voluntarios en las escuelas para los estudiantes con discapacidad, en actividades de conservación, interpretación, entre otras ${ }^{5}$.

Los viajes educativos se refieren a experiencias que pueden incluir una gran variedad de actividades tales como clases de lenguaje local, agroturismo, lecciones de artes y oficios tradicionales, por mencionar solo algunas. Ofrecen una gran cantidad de oportunidades para educadores, familias y viajeros individuales interesados en aprender más acerca de un tema o área geográfica y para aprender sobre el mundo a través de expertos locales.

\section{MARCO CONTEXTUAL}

El área cubierta por el Geoparque aspirante Huasteca Potosina se ubica en una de las cuatro regiones que conforman el estado de San Luis Potosí, México. El nombre de Huasteca Potosina se remonta a la época prehispánica. El líder nativo Cuexteca trajo a los primeros colonos a la parte oriental del país, arraigando así la cultura huasteca (la cultura del Caracol) también conocida como Teenek.

\footnotetext{
${ }^{3}$ The Importance of Scientific Travel. (n.d.). Retrieved from http://www.save-travel.org/travel-blog/why-scientific-travel/

${ }^{4}$ Making Memories Through Educational Travel. (n.d.). Retrieved from http://www.save-travel.org/travel-blog/making-memories-througheducational-travel/

${ }^{5}$ Andaman Discoveries. (n.d.). Retrieved from http://www.andamandiscoveries.com/volunteer-in-thailand/
} 
La cultura huasteca junto con los náhuatls y los pames aún mantienen sus tradiciones y costumbres prehispánicas y siguen sus vidas de la misma manera que siempre lo han hecho, convirtiendo a esta región en un área vibrante y mágica de México para ser descubierta y redescubierta, lo que le otorga un potencial para visitantes del segmento CAVE.

El Geoparque propuesto se encuentra en una parte de la provincia fisiográfica conocida como Sierra Madre Oriental y parte de la llanura costera del Golfo de México. Las coordenadas geográficas que delimitan el Geoparque son $21^{\circ} 27^{\prime}$ a $22^{\circ} 24^{\prime}$ de latitud norte a $98^{\circ} 19^{\prime}$ a $99^{\circ} 3^{\prime}$ de longitud oeste. El Geoparque incluye, de norte a sur, 6 municipios de los 58 que conforman el estado: Ébano, Tamuín, Ciudad Valles, Tamasopo, Aquismón y Huehuetlán. El perímetro total es 1.307,68 Km.

A continuación, en la Figura 1 se puede observar la ubicación.

Figura 1. Ubicación del Geoparque Aspirante Huasteca Potosina

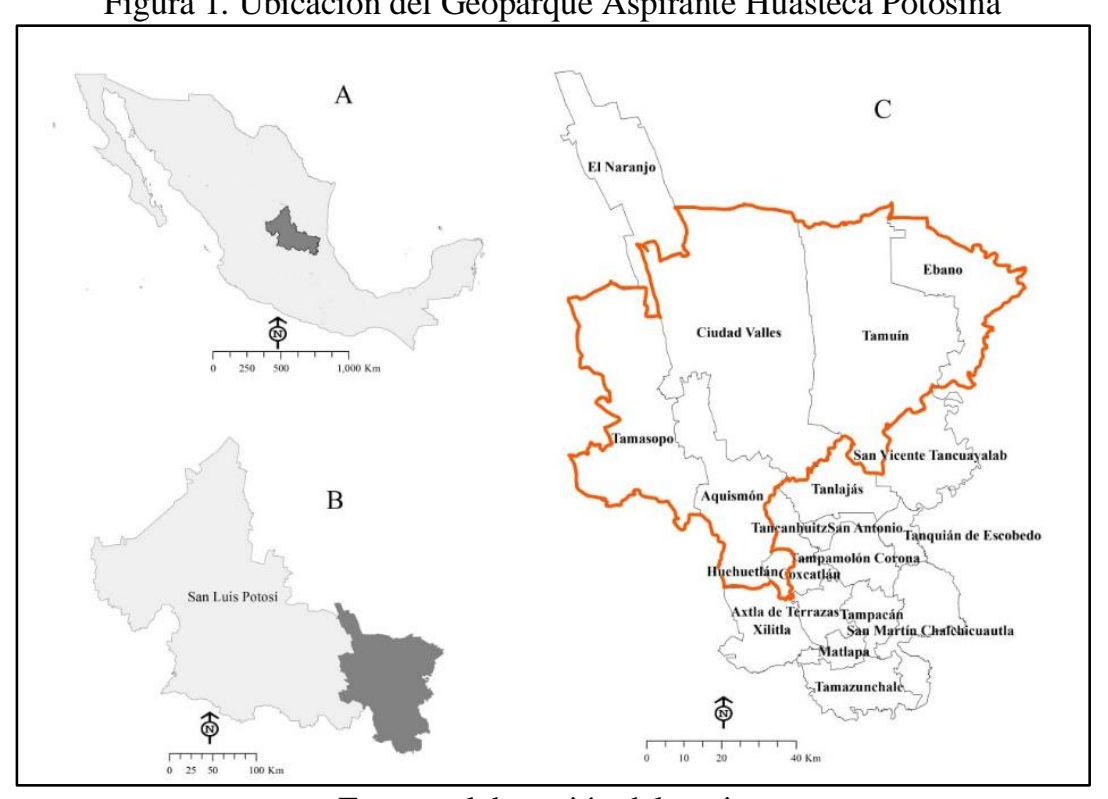

Fuente: elaboración del equipo

El área total del proyecto es de 7,153.97 km2. El territorio es grande en la superficie, pero es más importante debido a su patrimonio geológico, su diversidad cultural y natural y las oportunidades de desarrollo para las personas que viven en esta región abundante.

La posición geográfica de la zona es excepcional, en la frontera donde convergen las ecozonas neotropicales y neárticas. Cuenta con una importante geodiversidad, variedad de paisajes y una gran cantidad de recursos naturales. Es en lo que, históricamente, se ha clasificado como el área de transición, entre Mesoamérica y Aridoamérica y es el hogar de muchos asentamientos prehispánicos que le confieren una enorme importancia cultural y patrimonial.

El clima es altamente específico, lo que hace que la Huasteca Potosina sea única, con rangos desde un promedio de $26^{\circ}$ durante todo el año hasta máximos récord de $50^{\circ} \mathrm{C}$ en el mes de mayo. El clima es 
cálido y subhúmedo con abundantes precipitaciones en los meses de verano y una escasez total de lluvia de diciembre a mayo. Esto es en gran medida responsable de los bosques con escasa vegetación.

La población total del Geoparque, de acuerdo con el último censo (2015), era de 345,557 habitantes (12.7\% de la población total del Estado y 0.27\% del país). Desde 1930 hasta la fecha, existe una tasa constante de crecimiento de la población, lo que puede facilitar la planificación de políticas públicas centradas en el desarrollo de las comunidades locales.

Hoy en día los indígenas constituyen una sociedad multiétnica, plural, con identidades y culturas distintas, en las que el idioma, la historia y el territorio, el estilo de vida, el sistema cosmológico y la relación con la tierra los definen. El 94\% de los grupos indígenas del estado de San Luis Potosí viven en la región de la Huasteca y se hablan tres idiomas (Huasteco o Tennek, Náhuatl y Xi?úi). El 51.18\% de la población en el territorio son mujeres y el $48.81 \%$ son hombres; Esto indica que los esfuerzos deben centrarse en el empoderamiento de las mujeres.

La distribución del territorio es eminentemente rural y, desafortunadamente, es un territorio que todavía tiene condiciones de alta y muy alta marginación social. El proyecto tiene como objetivo ayudar a superar el retraso y mejorar la calidad de vida de sus habitantes.

La mitad de la población tiene menos de 26.16 y el $30 \%$ de la población total corresponde a niños menores de 15 años. Por lo tanto, consideramos que el Geoparque podría ofrecer una oportunidad significativa para las generaciones futuras de la región, mismas que pueden ser involucradas en proyectos turísticos para el mercado CAVE.

Los procesos migratorios son significativos en la región, normalmente como resultado de la falta de oportunidades percibida. El Geoparque pretende promover emprendimientos locales basados en la geodiversidad y el turismo CAVE, ofreciendo así a grupos vulnerables la posibilidad de permanecer en la región.

El empleo formal corresponde al $44.8 \%$ y el informal al $53.2 \%$, lo que se traduce en un desafío para el proyecto Geoparque, ya que es necesario promover las condiciones necesarias para reducir las cifras de informalidad laboral que se registran actualmente.

- La región de la Huasteca, desde 2012 presenta un aumento sostenido en la actividad turística. En 2018, la 1legada de turistas se registró en 300 mil visitantes. Las estadísticas indican que en marzo y abril existe una mayor demanda, lo que se traduce en una alta estacionalidad. Ello conlleva a la necesidad de crear productos innovadores, atractivos y diversificados para atraer la atención de los segmentos potenciales que visitan la región durante todo el año. Los seis motivos principales para viajar a la Huasteca Potosina desde 2010 hasta 2012 fueron aventura, naturaleza, ecoturismo, visita a familiares, cultura y religión. Este resultado puede atribuirse a la creciente preferencia de los viajeros por destinos emergentes menos conocidos, en vez de destinos maduros muy concurridos. En 2014 y 2015, la razón más frecuente para viajar fue el de vacaciones, que perfectamente pudo incluir motivos de aventura, naturaleza, cultura y actividades religiosas, así como visita a familiares y amigos. La propuesta del turismo CAVE representa una excelente oportunidad para ofrecer experiencias turísticas con interés científico o educativo basado en la geodiversidad, con su claro vínculo con la cultura y la naturaleza como es el caso de las rutas de Huehuetlán - Ruta del Viento y la Fertilidad; Aquismon Cuevas, Ríos y Cañadas, entre otros. 
Vale resaltar que existe un Centro de Interpretación del Geoparque en Ciudad Valles, ubicado en el Centro Cultural Huasteca que contiene los elementos necesarios para la difusión del patrimonio y la geoeducación. En otros municipios hay centros ceremoniales y centros de interpretación de la cultura y la naturaleza que podrían habilitarse para el desarrollo de espacios CAVE.

En el estado de San Luis Potosí existe una oferta de 501 hoteles, de los cuales 203 se encuentran en la huasteca potosina, siendo este el $40.52 \%$ de la oferta total. El 49,76\% se ubica en la región del proyecto con 100 hoteles distribuidos entre los 6 municipios.

Vale la pena señalar la ubicación estratégica del estado, que es el único en la República Mexicana que limita entre nueve estados: Coahuila, Nuevo León, Tamaulipas, Veracruz, Hidalgo, Querétaro, Guanajuato, Jalisco y Zacatecas, otorgando una cercanía con los principales mercados nacionales. En referencia a las carreteras, los Geositios tienen una muy buena accesibilidad, por carreteras estatales y federales, aunque todavía hay caminos de terracería.

Existe un aeropuerto nacional en el municipio de Tamuín, a $30 \mathrm{~km}$ de Ciudad Valles; este prevé una expansión y se prevé ofrecer vuelos internacionales a través de un proyecto por parte del Gobierno Federal. Desde la capital del Estado, el Geoparque está ubicado a $200 \mathrm{~km}$ de distancia, a $730 \mathrm{~km}$ de la Ciudad de México y a $180 \mathrm{~km}$ del Puerto de Tampico, que tienen aeropuertos internacionales.

Actualmente los sitios de atracción turística del Geoparque se promueven ampliamente y atraen a miles de visitantes que dinamizan una variada oferta de actividades turísticas. Están representados por la belleza natural del paisaje físico, principalmente asociado con sus componentes de carácter geológico y geomorfológico; la rica biodiversidad reflejada en una amplia diversidad de ecosistemas; los lugares con arquitectura colonial o religiosa de los siglos XVI, XVII y XVIII; la riqueza de un legado prehistórico y arqueológico singular; y las culturas vivas representadas por tres grupos étnicos diferentes que se distribuyen en diversos núcleos de población. En virtud de la riqueza patrimonial con la que cuenta se define como una región con extensa vocación turística, considerada muy atractiva para los turistas y visitantes, tanto de fuera como del interior del estado, y paulatinamente se ha ido consolidando como un destino importante en el ámbito del turismo.

\section{RESULTADOS}

Con más de 4 años de trabajo con autoridades estatales y municipales, actores sociales de la región, prestadores de servicios, voluntarios, académicos, tesistas, entre otros, además de visitas de campo, se logran identificar y reconocer 33 sitios de interés geológico - geositios. En la experiencia y labores de gabinete, fue posible señalar el potencial turístico natural dentro del territorio huasteco y, en particular, de los 6 municipios en estudio. Este levantamiento mostró la riqueza de geositios, paisajes, geoformas, fósiles, entre otros. 
En virtud de este rico patrimonio, la región tiene un gran potencial turístico tanto a nivel nacional como internacional. En el Proyecto Geoparque, hay cuatro geositios que se consideran de interés científico internacional y que están relacionadas con procesos de hidrología kárstica que afectan a la piedra caliza del Cretácico.

Dichos geositos han sido objeto de estudio científico y tienen un gran valor educativo debido a los procesos activos e inactivos que se pueden observar, interpretar y explicar in situ. Estos corresponden a las geoformas kársticas y dos de ellas se encuentran en áreas naturales protegidas.

Figura 2. Mapa del Geoparque Aspirante Huasteca Potosina

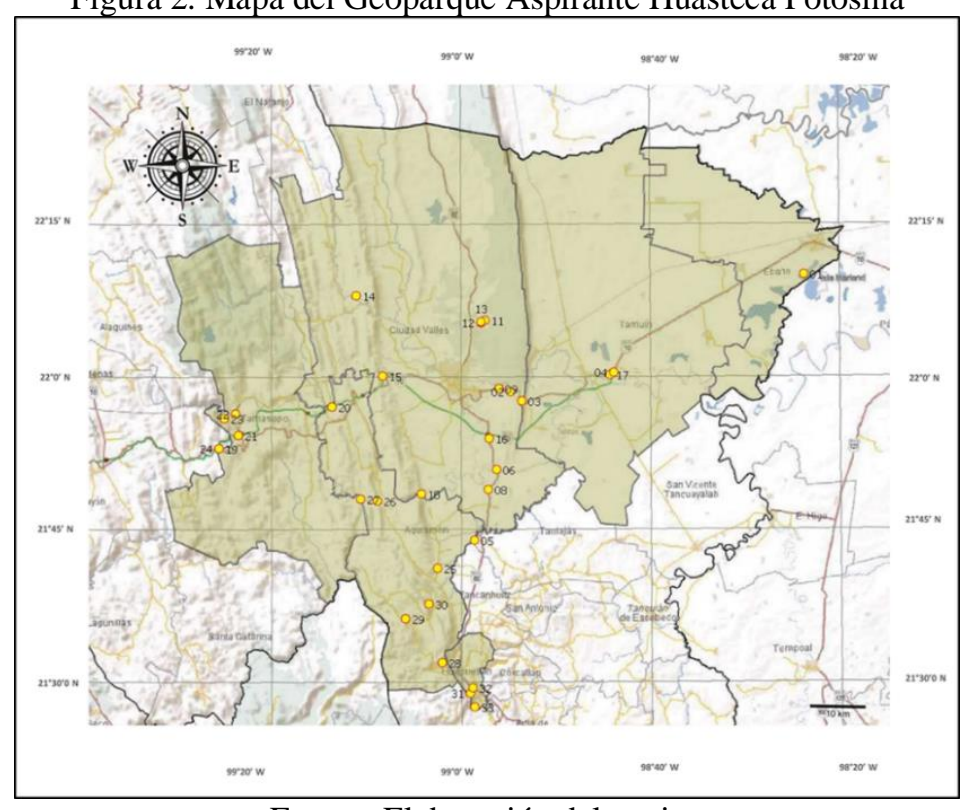

Fuente: Elaboración del equipo

Se han identificado 12 sitios naturales que están vinculados al patrimonio geográfico. Esos se interpretan con una visión holística que forma parte de los otros patrimonios. Se promueve a través del enlace con el geopatrimonio y las georutas que incluye visitar estos sitios de interés natural.

Se han identificado 10 muestras de patrimonio cultural en el territorio que son dignas de ser valoradas para el desarrollo de georutas que ayuden a llevar a cabo proyectos de turismo CAVE, al igual que el patrimonio inmaterial que revela la cosmogonía de los pueblos originarios de la región y tiene una declaración de la UNESCO como patrimonio inmaterial: los festivales de Xantolo, Zona Teenek y Ritual del Alba.

\subsection{METODOLOGÍA PARA LA EVALUACIÓN DEL TURISMO CAVE}

La evaluación, realizada por consultores estudiantiles de la GWU y la UASLP bajo la dirección de profesores de ambas universidades, es la culminación de una extensa investigación documental y de campo, así como de procesos participativos que incorporan y reflejan las percepciones, prioridades y 
desafíos expresados por los líderes locales en representación de los gobiernos local y estatal, el sector privado y la sociedad civil. Los resultados y recomendaciones se basaron en un análisis confiable del destino llevado a cabo en el transcurso de diez semanas a partir de la investigación.

Durante las dos semanas de trabajo de campo, los equipos realizaron las siguientes tareas:

- Una encuesta a más de 100 residentes de la comunidad

- Diecisiete (17) evaluaciones en profundidad de atractivos turísticos y sitios que se centraron en el desarrollo de productos turísticos, atractivos turísticos y gestión de sitios, así como temas de marketing de destinos

- Tres (3) talleres comunitarios, en los cuales se dio prioridad a los temas y se validaron recomendaciones

- Sesenta y seis (66) entrevistas con actores claves de la región

El trabajo realizado se tradujo en un total de 2 mil 500 horas de recopilación y análisis de datos y cubrió las siguientes fases:

- Fase 1: Investigación y desarrollo de enfoques

- Las actividades de inicio del proyecto incluyeron más de 100 horas de investigación documental, evaluaciones preliminares de productos turísticos y un plan integral para el trabajo de campo basado en la situación actual de la Huasteca Potosina de conformidad con los tres municipios. También se investigó mejores prácticas y lecciones aprendidas de otros casos de turismo comunitario tanto a nivel nacional como internacional con posibilidades de ser implementadas en las comunidades de los tres municipios.

- Fase 2: Despliegue del equipo para trabajo de campo y evaluaciones en los sitios

- El equipo consultor estudiantil fue dividido en tres equipos multidisciplinarios. Sus actividades incluyeron 17 evaluaciones pormenorizadas de los atractivos turísticos en cada uno de los tres sitios detallando las prioridades, necesidades y retos. El equipo realizó 66 entrevistas y reuniones con las partes interesadas, efectuó tres talleres comunitarios y totalizó 167 horas de recopilación de datos con actores del sector público y privado.

- Fase 3: Análisis y redacción del informe

- Los datos fueron analizados a fin de producir un informe integrado para la región, combinando las recomendaciones y directrices que entregarán un plan de acción concreto para las comunidades dentro del proyecto propuesto de Geoparque.

Los objetivos de la investigación fueron:

1. Evaluar las oportunidades y los retos asociados con el desarrollo de productos turísticos basados en la comunidad y la mejora en la gestión de atractivos turísticos y sitios ubicados en las comunidades de La Morena y Tanchachín, en el municipio de Aquismón, las comunidades de El Platanito, Micos y Teenek, en el municipio de Ciudad Valles, así como en el centro del Pueblo Mágico Xilitla y Las Pozas (Jardín Escultórico de Edward James), en el Municipio de Xilitla.

2. Evaluar el potencial de atraer y comprometer a los segmentos del mercado de turismo CAVE a la región.

3. Realizar propuestas innovadoras de Turismo CAVE para el Geoparque propuesto. 
A fin de poder identificar las potencialidades del Turismo CAVE, se realizó un estudio FODA de las Fortalezas, Oportunidades, Debilidades y Amenazas del territorio propuesto para el Geoparque, encontrando los siguientes resultados.

Figura 3. Cuadro del análisis FODA

\begin{tabular}{|c|c|}
\hline Fort & - Debilidades \\
\hline 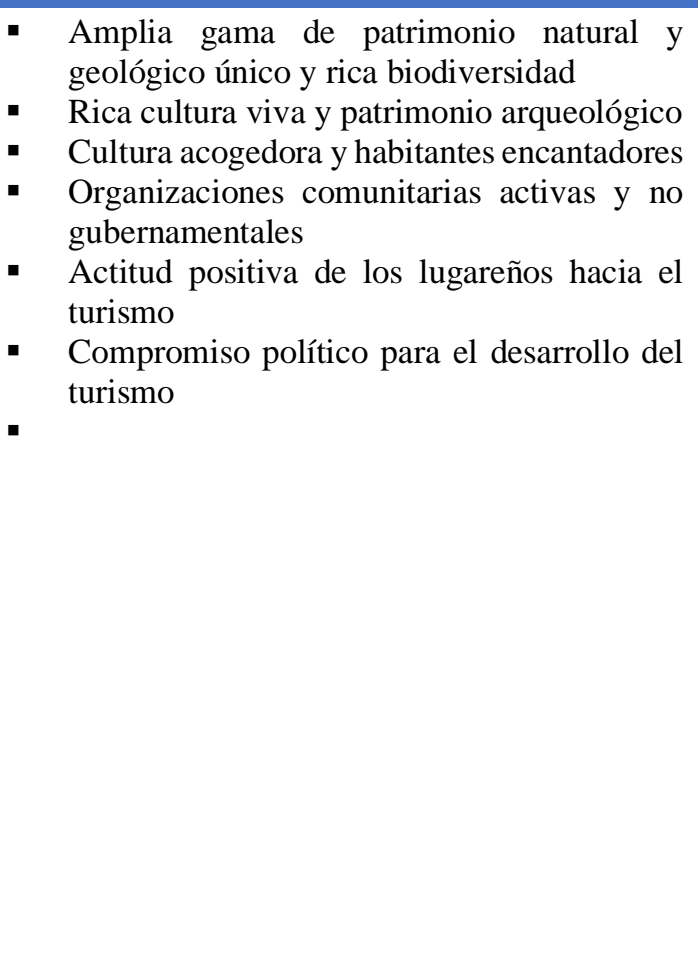 & $\begin{array}{l}\text { - Falta de coordinación de la gestión y el marketing como } \\
\text { destino } \\
\text { - Capacidad limitada de transporte aéreo } \\
\text { - Falta de confianza y colaboración entre las partes } \\
\text { interesadas } \\
\text { - Infraestructura deficiente (como el servicio a celulares e } \\
\text { internet) } \\
\text { - Daño en los sitios por las aglomeraciones en temporada } \\
\text { alta } \\
\text { - Dependencia de un solo mercado emisor para el turismo, } \\
\text { es decir, el mercado interno } \\
\text { Interpretación mínima (incluidas las señalizaciones) en } \\
\text { - Capitios y atractivos turísticos } \\
\text { - } \text { particular guías de turistas } \\
\text { Incongruencia al garantizar la seguridad y protección de } \\
\text { - Falta de gestión y control de los impactos en los atractivos } \\
\text { - turísticos } \\
\text { Disponibilidad limitada de información detallada en línea } \\
\text { sobre los productos turísticos } \\
\text { - }\end{array}$ \\
\hline - Opor & AII \\
\hline $\begin{array}{l}\text { - Oportunidades para diversificar la oferta de } \\
\text { productos turísticos basados en la riqueza de } \\
\text { la cultura } \\
\text { - Creación de empleos y aumento de las micro } \\
\text { y pequeñas empresas } \\
\text { Mayor conocimiento y valoración del } \\
\text { patrimonio natural, geológico y cultural a } \\
\text { nivel local y regional } \\
\text { - Nuevas inversiones del sector privado } \\
\text { - Desarrollo del mercado de turismo de nicho } \\
\text { - Participación de la población local en la } \\
\text { toma de decisiones y planeación acerca del } \\
\text { turismo comunitario }\end{array}$ & $\begin{array}{l}\text { - La "fragmentación" de la responsabilidad, sin mecanismos } \\
\text { de coordinación y colaboración } \\
\text { - Impactos ecológicos de las industrias extractivas locales, } \\
\text { como azúcar, ganado y minería en los ecosistemas del } \\
\text { Geoparque y el estilo de vida de la comunidad } \\
\text { - Actividades humanas no sustentables, como la caza } \\
\text { furtiva, deforestación y quema descontrolada } \\
\text { - Actos de saqueo y vandalismo en sitios arqueológicos } \\
\text { - Migración de jóvenes y otras personas de las grandes } \\
\text { - } \quad \text { Percepción de riesgos para la salud y la seguridad (carteles, } \\
\text { Zika, falta de ambulancias, distancia a los centros de salud, } \\
\text { entre otros) }\end{array}$ \\
\hline
\end{tabular}

Fuente: Elaboración del equipo

Con base en la evaluación realizada, el equipo consultor formuló las siguientes recomendaciones para desarrollar el segmento de mercado CAVE. 
Figura 4. Cuadro del análisis FODA.

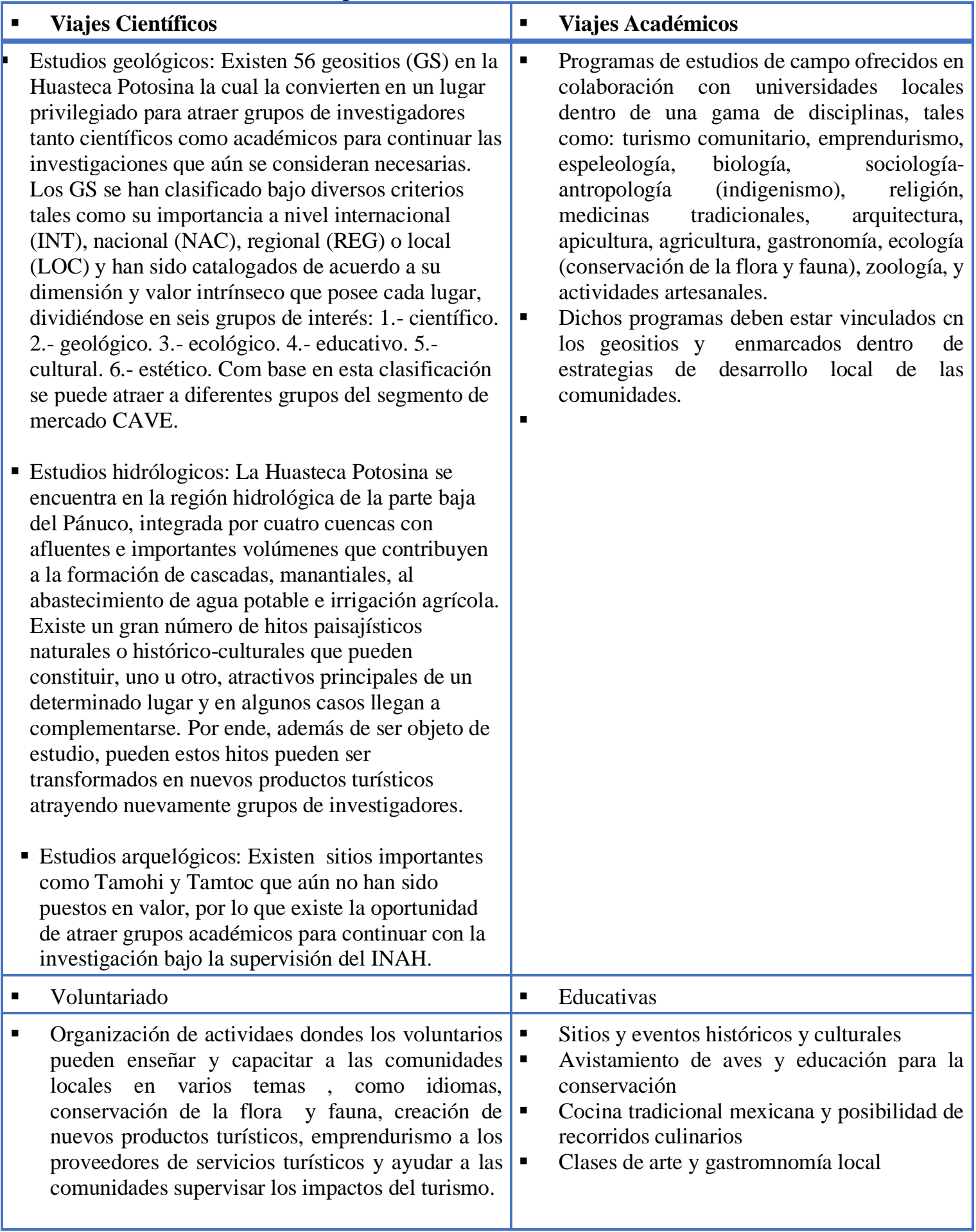

Fuente: Elaboración del equipo

Se proyecta la creación de un Centro de Turismo CAVE que conecte los potenciales sitios geoturísticos con los segmentos de mercado; que permita crear y administrar los productos que atraen a los mercados internacionales científicos, académicos, voluntarios y de educación; fomentar los beneficios económicos a largo plazo en el ámbito local y finalmente, el desarrollo de proyectos CAVE para ayudar 
a mejorar la experiencia del turismo local y crear oportunidades de desarrollo para el sector turístico y las comunidades locales.

El caso más reciente que ha permitido mostrar y acercar al conocimiento de la naturaleza geológica del territorio del proyecto Geoparque Huasteca Potosina fue la Primera Excursión Geologica "viajando a través del tiempo geológico" en un lapso comprendido desde hace 153 millones de años hasta la actualidad, visitando sitios con avistamientos de diversas estructuras correspondientes a la porción del borde oriental de la PVSLP (Plataforma Valles-San Luis Potosí) donde se depositaron rocas de ambiente de cuenca y plataforma en el Mesozoico y las cuales posteriormente fueron intensamente deformadas.

\section{CONCLUSIONES}

El mercado CAVE representa un segmento creciente de la industria mundial del turismo que difiere en varios aspectos clave de las formas más convencionales de turismo. Representa una amplia gama de turistas que viajan a un destino para dedicarse a la investigación, el aprendizaje o el turismo de voluntariado.

Por definición, aquellos atraídos al turismo CAVE suelen estar interesados en actividades que pueden aprovecharse fácilmente para impulsar la preservación del patrimonio natural y cultural, así como una mejoría en las condiciones de vida de los lugareños (SAVE Travel Alliance, s.f.).

Las actividades de CAVE afectan no solo a las economías locales, sino que también conllevan beneficios económicos a largo plazo a nivel nacional y local mediante el fomento de las capacidades de las instituciones educativas, científicas y culturales.

El territorio cubierto por el Geoparque aspirante tiene un potencial significativo para el desarrollo de actividades de Turismo CAVE, con miras a un turismo sostenible enfocado en resaltar el patrimonio geológico, la geodiversidad, la biodiversidad, la historia y la cultura de la huasteca potosina, lo que representa una oportunidad para la dinamización de la economía y desarrollo sostenible para las comunidades locales.

Tenemos claro que uno de los propósitos principales de un Geoparque Global de la UNESCO es poder identificar y crear los vínculos entre el patrimonio geológico y todos los demás aspectos de la naturaleza, patrimonio cultural e intangible y ponerlo en valor para el desarrollo de proyectos con las comunidades locales, por lo que el turismo CAVE se considera como una excelente herramienta para el empoderamiento de los habitantes de la región que estén interesados en diseñar y crear microemprendimientos basados en el estudio y divulgación del patrimonio geológico, ambiental y cultural de la región. 


\section{BIBLIOGRAFÍA}

García Canclini, N.2007. "Odios globalizados", Publicado en Cruz, Manuel, coordinador: "Odio, violencia, emancipación”, Editorial Gedisa, Barcelona, 2007.

Geoparques Mundiales de la UNESCO. 2017. Celebrando el Patrimonio de la Tierra, sosteniendo las comunidades locales. Publicado en 2017 por la Organización de las Naciones Unidas para la Educación, la Ciencia y la Cultura. Paris, France. http://www.unesco.org/open-access/terms-useccbysa-en

INEGI (Instituto Nacional de Estadística y Geografía), 2015. Estadísticas intercensales. Conteo de Población y Vivienda México.

Jafari, J. 2005. "El turismo como disciplina científica”, Política y Sociedad, 2005, Vol. 42 Núm. 1: 39-56, publicado en el portal de revistas científicas complutenses, Universidad Complutense de Madrid,23.02.05.

Márquez, A. R. y Sánchez, Á. 2007. Turismo y ambiente: la percepción de los turistas nacionales en Bahía de Banderas, Nayarit. México, Investigaciones Geográficas, 64(3), 134-152.

Márquez, D. 2004. “Turismo sostenible y desarrollo local”, documento integrante de la serie Pensamiento global para el desarrollo local; edita el Programa Delnet del Centro Internacional de Formación de la OIT, Turín, Italia.

Martinez, P. 2010. Identificación, caracterización y cuantificación de geositios, para la creación del I Geoparque en Chile, en torno al Parque Nacional Conguillío. Memoria para optar al título de Geólogo. Departamento de Geología Facultad de Ciencias Físicas y Matemáticas, Universidad de Chile. http://repositorio.uchile.cl/bitstream/handle/2250/103845/cfmartinez_pe.pdf?sequence=3

McLaren, D. 2003. "Rethinking Tourism \& ecotravel”, Kumarian Press Inc., Publication, 2003 ISBN: 1-56549-169-6

Meixueiro, G. 2006. Las cifras del turismo internacional en México. Socioscopio, 8, 46-55.

Meixueiro, G.2006. "Las cifras del turismo internacional en México", Revista de la Cámara de Diputados de México, Socioscopio núm. 8, Centro de Estudios Sociales y de Opinión Pública, México, 2006, Editorial Nueva Época.

Navarrete, F. 2008. Los pueblos indígenas de México [texto] / Federico Navarrete Linares - México : CDI, 2008.141 p. : mapas, tabs., fots. - (Pueblos Indígenas del México Contemporáneo) Incluye bibliografía ISBN 978-970-753-157-4

Organización Mundial del Turismo (OMT), Sitio oficial de la en la sección de turismo sostenible. http://www.unwto.org/sdt/index.php

SAVE TRAVEL ALLIANCE. (n.d.). Academic Travel is Invaluable Here's Why. Retrieved 10 4, 2016, from SAVE TRAVEL ALLIANCE: http://www.save-travel.org/travel-blog/academic-travel-is-invaluable-heres-why/

SAVE Travel Alliance. (n.d.). Making Memories Through Educational Travel. Retrieved 10 4, 2016, from SAVE Travel Alliance: http://www.save-travel.org/travel-blog/making-memories-through-educational-travel/

SAVE Travel Alliance. (n.d.). SAVE Campeche Mexico. Retrieved 10 4, 2016, from SAVE Travel Alliance: http://www.save-travel.org/destinations/campeche-mexico/about/

SAVE Travel Alliance. (n.d.). The Importance of Scientific Travel. Retrieved 10 4, 2016, from SAVE Travel Alliance: http://www.save-travel.org/travel-blog/why-scientific-travel/

Secretaria de turismo del estado de San Luis Potosí http://www.turismoslp.com.mx/

Secretaría de Turismo Federal, 2019. Resultados de la actividad turística 2019. Subsecretaría de Planeación y Política Turística. https://www.datatur.sectur.gob.mx/RAT/RAT-2019-12(ES).pdf 